\title{
Functional Acetabular Orientation Varies Between Supine and Standing Radiographs: Implications for Treatment of Femoroacetabular Impingement
}

\author{
James R. Ross MD, Eric P. Tannenbaum MD, Jeffrey J. Nepple MD, \\ Bryan T. Kelly MD, Christopher M. Larson MD, Asheesh Bedi MD
}

Published online: 6 January 2015

(C) The Association of Bone and Joint Surgeons (B) 2014

\begin{abstract}
Background Often, anteroposterior (AP) pelvic radiographs are performed with the patient positioned supine. However, this may not represent the functional position of the pelvis and the acetabulum, and so when assessing patients for conditions like femoroacetabular impingement (FAI), it is possible that standing radiographs better incorporate the dynamic influences of periarticular
\end{abstract}

\footnotetext{
One or more of the authors has received funding outside of submitted work from Smith \& Nephew (Andover, MA, USA) (JJN); Smith \& Nephew and A3 Surgical (La Tronche, France) (CML); and Smith \& Nephew, A3 Surgical, and Pivot (Sunnyvale, CA, USA) (BTK, AB). During the study period, an amount less than USD 10,000 was received. One or more of the authors has stock/stock options in A3 Surgical (CML, AB) and A3 Surgical and Pivot (BTK).

All ICMJE Conflict of Interest Forms for authors and Clinical Orthopaedics and Related Research ${ }^{\circledR}$ editors and board members are on file with the publication and can be viewed on request.

Clinical Orthopaedics and Related Research ${ }^{\mathbb{R}}$ neither advocates nor endorses the use of any treatment, drug, or device. Readers are encouraged to always seek additional information, including FDA-approval status, of any drug or device prior to clinical use. Each author certifies that his or her institution approved the human protocol for this investigation, that all investigations were conducted in conformity with ethical principles of research, and that informed consent for participation in the study was obtained.
}

\section{J. R. Ross}

Broward Orthopedic Specialists, Fort Lauderdale, FL, USA

E. P. Tannenbaum, A. Bedi $(\bowtie)$

Sports Medicine and Shoulder Service, Department of Orthopaedic Surgery, University of Michigan, MedSport, 24 Frank Lloyd Wright Drive, Ann Arbor, MI 48106, USA e-mail: abedi@umich.edu; abedi@med.umich.edu

\section{J. J. Nepple}

Department of Orthopaedic Surgery, Washington University in St Louis, St Louis, MO, USA musculature and sagittal balance. However, this thesis remains largely untested.

Questions/purposes The purpose of this study was to determine the effect of supine and standing pelvic orientation on (1) measurements of acetabular version and common radiographic signs of FAI as assessed on two- and three-dimensional (3-D) imaging; and (2) on terminal hip range of motion (ROM).

Methods Preoperative pelvic CT scans of 50 patients (50 hips) who underwent arthroscopic surgery for the treatment of FAI between July 2013 and October 2014 were analyzed. The mean age of the study population was $29 \pm 10$ years (range, $15-50$ years) and $70 \%$ were male. All patients had a standing AP pelvis radiograph, a reconstructed supine radiograph from the CT data, and a 3-D model created to allow manipulation of pelvic tilt and simulate ROM to osseous contact. Acetabular version was measured and the presence of the crossover sign, prominent ischial spine sign, and posterior wall sign were recorded on simulated plain radiographs. Measurements of ROM to bony impingement were made during (1) simulated hip flexion; (2) simulated internal rotation in $90^{\circ}$ of flexion (IRF); and (3) simulated internal rotation in $90^{\circ}$ of flexion and $15^{\circ}$ adduction (FADIR), and the location of bony

\author{
B. T. Kelly, A. Bedi \\ Hospital for Special Surgery, New York, NY, USA \\ C. M. Larson \\ Minnesota Orthopedic Sports Medicine Institute at Twin Cities \\ Orthopedics, Edina, MN, USA
}


contact between the proximal femur and acetabular rim was defined. These measurements were calculated for supine and standing pelvic orientations. A paired Student's t-test was used for comparison of continuous variables, whereas chi square testing was used for categorical variables. A $p$ value of $<0.05$ was considered significant.

Results When changing from supine to the standing radiographs, both mean cranial and central version increased by $2^{\circ} \pm 4^{\circ}\left(95 \%\right.$ confidence interval $\left.[\mathrm{CI}], 1^{\circ}-3^{\circ}\right)$ and $2^{\circ} \pm 3^{\circ}\left(95 \% \mathrm{CI}, 1^{\circ}-3^{\circ}\right)$, respectively (both $\mathrm{p}<0.001)$. However, with the numbers available, there were no changes in the proportion of positive crossover, posterior wall, and prominent ischial spine signs. Standing pelvic position tilt resulted in an increased hip flexion of $3^{\circ}$ $\left(95 \% \mathrm{CI}, 2^{\circ}-4^{\circ}\right)$ as well as an increase in IRF of $2^{\circ}(95 \%$ CI, $\left.1^{\circ}-3^{\circ}\right)$ and FADIR of $3^{\circ}\left(95 \%\right.$ CI, $\left.2^{\circ}-4^{\circ}\right)$ (all $\mathrm{p}<0.001$ ).

Conclusions The functional orientation of the acetabulum varies between supine and standing radiographs and must be considered when diagnosing and treating patients with symptomatic FAI. Standing pelvic orientation results in posterior pelvic tilt and later occurrence of FAI in the arc of motion. Although we cannot recommend standing radiographs on the current study alone, we do recommend larger studies to determine whether any significant differences truly exist.

Level of Evidence Level III, diagnostic study.

\section{Introduction}

Femoroacetabular impingement (FAI), a common cause of hip pain in young, active adults, results from abnormal bony contact between the proximal femur and acetabulum during ROM. Radiographic evaluation is a critical component of the diagnostic evaluation and treatment decisionmaking process in these patients. An understanding of the effect of pelvic tilt, defined as the angle between the line connecting the midpoint of the sacral plate to the axis of the femoral heads and the vertical axis [13], on the appearance of the hip on pelvic radiographs is important when assessing young adults who present with hip pain $[5-8,19,21,22]$. Pelvic tilt is a natural component of the patient's posture and may vary substantially among patients, between sexes, and during different activities of daily living and sport-specific activities [8, 19]. The sacrococcygeal distance (SCD) has become an accepted surrogate measurement on the AP pelvic radiograph to assess pelvic tilt.

Often, AP pelvic radiographs are performed with the patient positioned supine. However, this may not represent the functional position of the pelvis and the acetabulum, and so when assessing patients for conditions such as FAI, it is possible that standing radiographs may better incorporate the dynamic influences of periarticular musculature and sagittal balance [1]. However, to our knowledge, no studies have evaluated the effect of weightbearing (standing) radiographs on the common radiographic measurements used assessing patients with FAI.

The purpose of this study was to determine the effect of supine and standing pelvic orientation on acetabular version parameters on two- and three-dimensional (3-D) imaging studies. Second, we aimed to compare the effect of these two orientations on simulated impingement-free terminal hip ROM.

\section{Patients and Methods}

Between July 2013 and October 2014, a single surgeon (AB) performed 66 arthroscopic FAI procedures; of those, 50 hips (76\%) in 50 patients presenting with symptomatic FAI underwent a standing AP pelvis and a preoperative CT scan and were treated with arthroscopic hip surgery for symptomatic FAI. The 16 patients (24\%) who were not included within the study did not have a standing AP pelvis radiograph. All patients who are indicated for arthroscopic treatment of FAI by the treating surgeon undergo a preoperative CT scan for surgical planning. All patients were diagnosed with FAI on the basis of symptoms and physical examination findings, which were confirmed with corresponding radiographic pathomorphology on plain films and 3-D imaging. The mean maximum CT-derived alpha angle for all hips was $73^{\circ} \pm 12^{\circ}$ (range, $49^{\circ}-96^{\circ}$ ) and was located on average at the 1:00 clockface position. The mean alpha angles at 12:00, 1:30, and 3:00 were $53^{\circ}, 68^{\circ}$, and $51^{\circ}$, respectively. Twenty-five hips (50\%) presented with isolated cam impingement, one hip (2\%) with isolated pincer impingement, and 24 hips (48\%) with combined cam and pincer pathomorphology. No other hip pathologies were noted such as Perthes or slipped capital femoral epiphysis. The mean age of patients in this series was $29 \pm 10$ years (range, 15-50 years). Seventy percent of the patients $(\mathrm{n}=35)$ were male, and $54 \%(\mathrm{n}=27)$ of the surgeries involved the right hip. This study was performed under an institutional review board-approved protocol.

In addition to standing AP pelvis and modified Dunn lateral radiographs performed with a fixed protocol, the patients underwent high-resolution CT scans of the pelvis (and distal femur for assessment of femoral version) as part of their clinical care and preoperative surgical planning. A modified CT protocol using a decreased radiation exposure of $3 \mathrm{mSv}$ was used to maximize patient safety as described in Milone et al. [16]. Positioning of the patient in the scanner was standardized with the legs in native abduction/ adduction and the patellae pointing directly anterior. The 
patient was positioned supine with a natural resting pelvic position. This resting, pelvic position was considered the patient's "supine" pelvic tilt.

The SCD [19, 21] was measured on the standing AP pelvic radiograph by two independent observers (JRR, EPT), on three separate occasions and the averages calculated. The preoperative CT scans were uploaded into a CT-based, computer software program (DYONICS PLAN; Smith \& Nephew, Andover, MA, USA) to generate patientspecific 3-D models of the hip. This software program also allowed generation of virtual plain radiographs, including a simulated AP pelvic radiograph. The "supine" SCD was calculated from the CT scan (Fig. 1A) with assistance of the software measurement tools. These virtual radiographs were also analyzed for parameters of acetabular and pelvic orientation. Static radiographic parameters (two-dimensional and 3-D) and dynamic ROM measurements to impingement were calculated for two pelvic positions: supine and standing (Fig. 1).

The dynamic software also allowed manipulation of the pelvic tilt and to create and analyze the standing pelvic orientation, the pelvic tilt of the virtual pelvis was adjusted until the CT-based SCD matched the measured SCD from the standing AP pelvic radiograph (Fig. 1B-C). SCD was defined as "appropriate" if 20 to $40 \mathrm{~mm}$ in male patients and 20 to $55 \mathrm{~mm}$ in female patients, as described by Tannast et al. [21].

Two-dimensional radiographic parameters, including the presence or absence of the crossover [18], prominent ischial spine [10, 11], and posterior wall signs [18], were determined. Additionally, the lateral center-edge angle (LCEA) [24] was measured. Three-dimensional radiographic parameters measured included acetabular version measurements at the 1:30 (cranial) position and 3:00 (central) position [15] as well as percent acetabular coverage at the anterior, superior, and posterior aspects of the acetabulum.

Simulated hip ROM to the point of osseous impingement was performed with the 3-D-generated model as previously described [2, 3] with the pelvis in the orientation of the supine and standing positions. No clinical ROM measurements were made in this series, because we measured the differences and changes in the ROM between the two various pelvic positions using the 3-D-generated models. The pelvis was fixed in the predefined position and the femur was free to move in all directions but constrained to rotate about the proscribed rotation axis, against the congruous acetabular surface. A posteriorly and superiorly directed force was applied to the femur to maintain reduction of the femur during simulation [3]. The femur was positioned with the posterior femoral condylar axis parallel to the horizontal axis of the pelvis (native femoral version). During the simulated ROM maneuvers, the femur was moved in a specific motion until contact between the femur and acetabulum occurred (detected by the resultant translation of the femoral head). This point of collision was defined as the occurrence of mechanical impingement, which was recorded in degrees of motion. Three ROM simulations were performed: (1) internal rotation in $90^{\circ}$ of hip flexion (IRF); (2) internal rotation in $90^{\circ}$ of hip flexion with $15^{\circ}$ of adduction (FADIR); and (3) maximum hip flexion. The location of contact on both the proximal femur and the acetabular rim was determined using standardized clockface nomenclature. The clockface was standardized between hips so that 12:00 was proximal and 3:00 was always anterior in both right and left hips [4, 14, 17].

\section{Statistics}

Statistical analysis was performed with Microsoft (Redmond, WA, USA) Excel software to compare the changes in radiographic parameters and ROM to impingement between the different pelvic tilt conditions. A paired Student's t-test was used for comparison of continuous variables, whereas chi square testing was used for categorical variables. Analysis was also performed to identify correlations between the radiographic measurements and signs of acetabular version and the change in pelvic tilt and SCD between supine and standing radiographs using the Mann-Whitney U-test for categorical variables and Pearson correlation for continuous variables. A $p$ value of $<0.05$ was considered significant.

\section{Results}

When moving from the supine to the standing position, both mean cranial acetabular version and central version increased by $2^{\circ} \pm 4^{\circ}\left(95 \%\right.$ confidence interval [CI], $\left.1^{\circ}-3^{\circ}\right)$ and $2^{\circ} \pm 3^{\circ}\left(95 \% \mathrm{CI}, 1^{\circ}-3^{\circ}\right)$, respectively $(\mathrm{p}<0.001$; Table 1). There was, however, no significant change in the percentage of positive radiographic signs of acetabular retroversion, namely crossover sign (44\% in supine versus $32 \%$ in standing; $\mathrm{p}=0.21)$, posterior wall sign ( $46 \%$ in supine versus $36 \%$ in standing; $\mathrm{p}=0.31)$, and prominent ischial spine sign ( $16 \%$ in supine versus $20 \%$ in standing; $p=0.60$ ). However, $27 \%$ (six of 22) of patients with a crossover sign in the supine position lost the appearance of this sign when in the standing position. There was no change in the LCEA $\left(32^{\circ} \pm 6^{\circ}\right.$ in supine versus $31^{\circ} \pm 6^{\circ}$ in standing; $p=0.84$ ). Additionally, the mean SCD significantly decreased from $35 \pm 14 \mathrm{~mm}$ to $28 \pm 18 \mathrm{~mm}(\mathrm{p}<0.001$; Table 1$)$. This pelvic orientation was created by manipulation of the pelvic tilt by a mean of $3^{\circ} \pm 5^{\circ}$ in the posterior direction (range, $16^{\circ}$ posterior to $10^{\circ}$ anterior). Seventy-four percent (37 of 50) of patients were noted to have appropriate SCD in the supine position. Sixty percent (30 of 50) of patients were noted to 


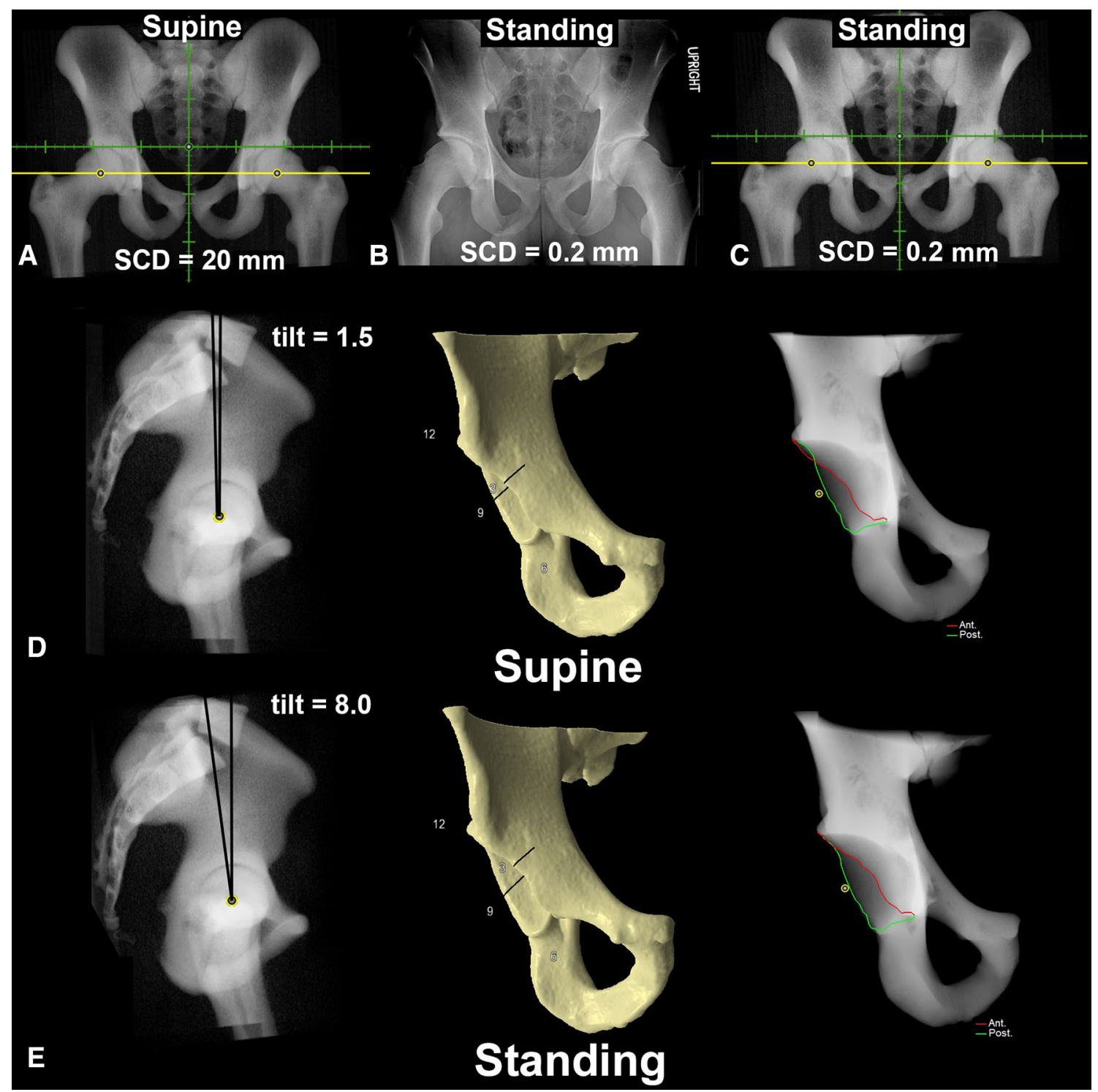

Fig. 1A-E Patient example demonstrating the supine virtual radiograph (A) reconstructed from the CT scan. (B) The standing AP pelvic radiograph demonstrated a $\mathrm{SCD}$ of $0.2 \mathrm{~mm}$. The standing virtual

have appropriate SCD in the standing position. A positive crossover sign on the standing radiograph was associated with a smaller change in pelvic tilt $\left(0.1^{\circ}\right.$ anterior versus $4.1^{\circ}$ posterior; $\mathrm{p}=0.015$ ). Similarly, a positive posterior wall sign on standing radiographs was also associated with a smaller change in pelvic tilt $\left(1^{\circ}\right.$ versus $\left.4^{\circ} ; \mathrm{p}=0.007\right)$. Additionally, hips that demonstrated less anteversion, more anterior coverage, and less posterior coverage displayed smaller differences in pelvic tilt between supine and standing radiographs $(\mathrm{p}<0.05)$.

A standing pelvic position resulted in an increase in hip flexion of $3^{\circ}\left(95 \% \mathrm{CI}, 2^{\circ}-4^{\circ}\right)\left(118^{\circ} \pm 16^{\circ}\right.$ for supine and $121^{\circ} \pm 16^{\circ}$ for standing; $\left.\mathrm{p}<0.001\right)$ when compared with the supine pelvic position. Similarly, there was also an radiograph (C) was created by increasing posterior pelvic tilt (D-E) by $6.5^{\circ}$. (D) Supine pelvic radiograph demonstrated a positive crossover sign, which is eliminated with standing radiographic evaluation $(\mathbf{E})$.

increase in internal rotation in flexion of $2^{\circ}(95 \% \mathrm{CI}$, $\left.1^{\circ}-3^{\circ}\right)\left(28^{\circ} \pm 14^{\circ}\right.$ for supine and $30^{\circ} \pm 14^{\circ}$ for standing; $\mathrm{p}<0.001)$ with an anterior shift in the location of the acetabular impingement (1:15 versus $1: 30 ; \mathrm{p}=0.05)$ (Table 2). The standing position also resulted in a $3^{\circ}(95 \%$ CI, $\left.2^{\circ}-4^{\circ}\right)$ increase in FADIR $\left(20^{\circ} \pm 14^{\circ}\right.$ for supine and $22^{\circ} \pm 14^{\circ}$ for standing; $\left.p<0.001\right)$ with a lateral shift in the femoral impingement ( $3: 00$ versus $3: 15 ; \mathrm{p}=0.01)$.

\section{Discussion}

Analysis and identification of acetabular morphology are critical in the decision-making process when evaluating 
Table 1. Two- and three-dimensional acetabular measurements in the supine and standing pelvic positions

\begin{tabular}{|c|c|c|c|c|c|}
\hline \multirow[t]{2}{*}{ Radiographic measurement } & \multicolumn{2}{|l|}{ Supine } & \multicolumn{2}{|l|}{ Standing } & \multirow[t]{2}{*}{$\mathrm{p}$ value } \\
\hline & Mean \pm SD & Range & Mean \pm SD & Range & \\
\hline Sacrococcygeal distance & $35 \pm 14 \mathrm{~mm}$ & $12-70 \mathrm{~mm}$ & $28 \pm 18 \mathrm{~mm}$ & -10 to $72 \mathrm{~mm}$ & $<0.001$ \\
\hline LCEA & $31^{\circ} \pm 6^{\circ}$ & $20^{\circ}-49^{\circ}$ & $32^{\circ} \pm 6^{\circ}$ & $20^{\circ}-46^{\circ}$ & 0.84 \\
\hline Cranial (1:30) acetabular version & $4^{\circ} \pm 10^{\circ}$ & $-19^{\circ}$ to $39^{\circ}$ & $6^{\circ} \pm 11^{\circ}$ & $-23^{\circ}$ to $33^{\circ}$ & $<0.001$ \\
\hline Mid (3:00) acetabular version & $16^{\circ} \pm 7^{\circ}$ & $0^{\circ}-30^{\circ}$ & $17^{\circ} \pm 8^{\circ}$ & $-1^{\circ}$ to $37^{\circ}$ & $<0.001$ \\
\hline Positive crossover sign & $44 \%$ & & $32 \%$ & & 0.21 \\
\hline Positive posterior wall sign & $46 \%$ & & $36 \%$ & & 0.31 \\
\hline Positive prominent ischial spine sign & $16 \%$ & & $20 \%$ & & 0.60 \\
\hline Anterior coverage & $33 \% \pm 4 \%$ & $28 \%-44 \%$ & $32 \% \pm 4 \%$ & $22 \%-43 \%$ & $<0.0001$ \\
\hline Superior coverage & $60 \% \pm 4 \%$ & $54 \%-70 \%$ & $59 \% \pm 4 \%$ & $53 \%-69 \%$ & 0.0001 \\
\hline Posterior coverage & $46 \% \pm 4 \%$ & $37 \%-57 \%$ & $47 \% \pm 5 \%$ & $38 \%-59 \%$ & $<0.0001$ \\
\hline
\end{tabular}

LCEA $=$ lateral center-edge angle.

Table 2. ROM to impingement and the corresponding femoral and acetabular impingement locations

\begin{tabular}{llllr}
\hline Dynamic motion & $\begin{array}{l}\text { Supine } \\
\text { Mean } \pm \mathrm{SD}\end{array}$ & $\begin{array}{l}\text { Standing } \\
\text { Mean } \pm \mathrm{SD}\end{array}$ & Change & Mean value \\
\cline { 3 - 5 } & $118^{\circ} \pm 16^{\circ}$ & $121^{\circ} \pm 16^{\circ}$ & $3^{\circ}$ & $-7^{\circ}$ to $18^{\circ}$ \\
Flexion & $5: 00$ & $5: 00$ & & 0.78 \\
Femoral impingement & $1: 45$ & $1: 45$ & $2^{\circ}$ & $-6^{\circ}$ to $10^{\circ}$ \\
Acetabular impingement & $28^{\circ} \pm 14^{\circ}$ & $30^{\circ} \pm 14^{\circ}$ & 0.42 \\
IRF & $2: 45$ & $2: 45$ & & 0.001 \\
Femoral impingement & $1: 15$ & $1: 30$ & $3^{\circ}$ & 0.05 \\
Acetabular impingement & $20^{\circ} \pm 14^{\circ}$ & $22^{\circ} \pm 14^{\circ}$ & $-8^{\circ}$ to $18^{\circ}$ \\
FADIR & $3: 15$ & $3: 00$ & & 0.001 \\
Femoral impingement & $1: 45$ & $1: 45$ & & 0.84 \\
Acetabular impingement & & &
\end{tabular}

$\mathrm{IRF}=$ internal rotation in $90^{\circ}$ of flexion; FADIR $=$ simulated internal rotation in $90^{\circ}$ of flexion and $15^{\circ}$ of adduction.

patients who present with hip pain and defining the most appropriate joint preservation treatment options $[9,12,20]$. Previous studies have demonstrated the effect of alterations in pelvic tilt on plain radiographic parameters of acetabular morphology $[6-8,19,21,22]$. AP pelvis radiographs are used routinely and are generally obtained with the patient positioned supine. Some have questioned the validity of supine pelvic radiographs in the patient with young hip disorders, because this pelvic position is not functional and does not include the dynamic influence of periarticular musculature and sagittal balance in the standing position [25]. The purpose of this study was to determine the effect of supine and standing pelvic orientation on acetabular version parameters on two- and 3-D imaging studies. Additionally, we aimed to compare the effect of these two orientations on simulated impingement-free terminal hip ROM.

The current study should be interpreted in light of its limitations. ROM simulations in the study include only bony morphology, ignoring contributions of labrum, cartilage, capsule, and periarticular soft tissue structures. This is reflected in the high level of IRF (mean of $28^{\circ}$ ) present in this FAI population in the supine position. Current technology does not allow for the inclusion of soft tissue structures. The pelvic position was also fixed in the simulations during ROM and although changes in dynamic pelvic tilt do likely occur during ROM, these changes are currently poorly understood and are an appropriate target for future research. Additionally, the CT scans were performed in the supine position and the pelvic position was manipulated to match the position of a standing radiograph. Although this manipulation is not an exact replica of the standing position, the software program allows measurement of the SCD and manipulation of the pelvic tilt to match the standing pelvic radiograph. Furthermore, femoral orientation is likely to influence ROM and was standardized with the posterior femoral condylar axis parallel to the horizontal axis of the pelvis. Although this orientation may not represent physiologic orientation, it was uniform between the pelvic orientations and by using a 
matched-pair study design, this is minimized. The current study included only patients with underlying FAI and the findings may not be applicable to those without such deformity. However, the vast majority of patients presenting with symptomatic labral tears have evidence of underlying FAI pathomorphology [23]. Finally, all patients within the study underwent surgical treatment of FAI and thus did not include patients who were treated without surgery. However, exposing these patients to the radiation of a CT scan, although limited, would be difficult to justify.

In our study, we demonstrate that the functional orientation of the acetabulum varies between supine and standing radiographs and should be considered when analyzing the radiographs of patients with symptomatic FAI. The SCD, which has become a commonly used surrogate measure of pelvic tilt, was noted to decrease by $7 \mathrm{~mm}$ when changing from the supine to standing position, which was slightly less than recently reported measurements by Pullen et al. [25], which noted a mean decrease of $11 \mathrm{~mm}$ among males and $16 \mathrm{~mm}$ among females. Troelsen et al. [26] also demonstrated similar changes in pelvic tilt when comparing supine with standing radiographs in a series of patients with acetabular dysplasia. Previous studies have defined "appropriate" values of the SCD; however, according to our study, between $26 \%$ and $40 \%$ of patients had a sacrococcygeal measurement outside of the previously established "appropriate" values [21]. This further agrees with the argument that pelvic position is variable among patients and must be taken into consideration when analyzing and treating patients with prearthritic hip pain. Similar to the study by Troelsen et al. [26], our study did not demonstrate any significant change in the LCEA. Other studies, however, have demonstrated significant decreases in the LCEA between $1.2^{\circ}$ and $3.6^{\circ}[8,25]$. These studies, however, performed measurements of true weightbearing radiographs and perhaps weightbearing through the hip may allow for slight femoral head lateral translation, which would result in a decrease in the LCEA measurement. Although we did not demonstrate any significant change in the radiographic markers of acetabular version (crossover, posterior wall, and prominent ischial spine signs), we did demonstrate a significant change in both the cranial and central version, which has not been investigated previously. It is perhaps that the study is underpowered to detect a radiographic difference in the signs of acetabular version given that $27 \%$ of patients with a crossover sign in the supine position ended up losing their crossover sign when changing to the standing position. Although $73 \%$ of patients did not demonstrate a change in this indicator of acetabular retroversion, this concept is important to note given that a false perception of focal cranial retroversion may lead to errant rim resection and risk of iatrogenic dysplasia. The role of dynamic and static alterations in pelvic tilt in FAI is poorly understood. In addition to the effect of acetabular parameters that were previously mentioned, our study also demonstrates small but significant changes in secondary terminal hip ROM to impingement when comparing the supine and standing pelvic positions. Although these mean values may not be clinically significant, the wide range of dynamic changes with pelvic alteration between supine and standing is not insignificant. Although the majority of patients in our study had a posterior tilt of the pelvis in the standing position, there were also patients within our study who had anterior pelvic tilt in the standing position, represented by an increase in the SCD. Within these patients, the standing position was accompanied by retroversion of the acetabulum and subsequent loss of simulated hip motion with the pelvis positioned anteriorly to match the standing SCD. Additionally, patients with more retroversion in the standing position have a lesser magnitude of pelvic mobility. This highlights the variability between patients and further emphasizes the concept of dynamic muscular control of the pelvis with resultant changes in pelvic tilt, which might compensate for impingement in some instances. Future studies, however, will need to validate whether these findings are also important clinically through both clinical measurements of ROM as well as correlating with patient-reported outcome scores.

In summary, we found that the functional orientation of the acetabulum varies between supine and standing radiographs and must be considered when diagnosing and treating patients with symptomatic FAI. We demonstrate pelvic tilt to be variable between patients with the standing pelvic orientation, on average, resulting in posterior pelvic tilt and thus a relative anteversion of the acetabulum and later occurrence of FAI in the arc of simulated motion. It is however also important to note that some patients demonstrated anterior pelvic tilt when moving from a supine to standing position. Although the common radiographic measures of acetabular version were not significantly different between groups, functional changes of the acetabulum do occur. Although we cannot recommend standing radiographs on the current study alone, we do recommend larger studies to determine whether any significant differences truly exist.

\section{References}

1. Babisch JW, Layher F, Amiot LP. The rationale for tilt-adjusted acetabular cup navigation. J Bone Joint Surg Am. 2008;90: 357-365.

2. Bedi A, Dolan M, Hetsroni I, Magennis E, Lipman J, Buly R, Kelly BT. Surgical treatment of femoroacetabular impingement improves hip kinematics: a computer-assisted model. Am J Sports Med. 2011;39(Suppl):43S-49S.

3. Bedi A, Dolan M, Magennis E, Lipman J, Buly R, Kelly BT. Computer-assisted modeling of osseous impingement and resection in femoroacetabular impingement. Arthroscopy. 2012;28: 204-210. 
4. Blankenbaker DG, De Smet AA, Keene JS, Fine JP. Classification and localization of acetabular labral tears. Skeletal Radiol. 2007;36:391-397.

5. Clohisy JC, Carlisle JC, Beaule PE, Kim YJ, Trousdale RT, Sierra RJ, Leunig M, Schoenecker PL, Millis MB. A systematic approach to the plain radiographic evaluation of the young adult hip. J Bone Joint Surg Am. 2008;90(Suppl 4):47-66.

6. Dandachli W, Ul Islam S, Richards R, Hall-Craggs M, Witt J. The influence of pelvic tilt on acetabular orientation and cover: a three-dimensional computerised tomography analysis. Hip Int. 2013;23:87-92.

7. Dandachli W, Ul Islam S, Tippett R, Hall-Craggs MA, Witt JD. Analysis of acetabular version in the native hip: comparison between 2D axial CT and 3D CT measurements. Skeletal Radiol. 2011;40:877-883.

8. Fuchs-Winkelmann S, Peterlein CD, Tibesku CO, Weinstein SL. Comparison of pelvic radiographs in weightbearing and supine positions. Clin Orthop Relat Res. 2008;466:809-812.

9. Ganz R, Gill TJ, Gautier E, Ganz K, Krugel N, Berlemann U. Surgical dislocation of the adult hip a technique with full access to the femoral head and acetabulum without the risk of avascular necrosis. J Bone Joint Surg Br. 2001;83:1119-1124.

10. Kakaty DK, Fischer AF, Hosalkar HS, Siebenrock KA, Tannast M. The ischial spine sign: does pelvic tilt and rotation matter? Clin Orthop Relat Res. 2010;468:769-774.

11. Kalberer F, Sierra RJ, Madan SS, Ganz R, Leunig M. Ischial spine projection into the pelvis: a new sign for acetabular retroversion. Clin Orthop Relat Res. 2008;466:677-683.

12. Kim WY, Hutchinson CE, Andrew JG, Allen PD. The relationship between acetabular retroversion and osteoarthritis of the hip. J Bone Joint Surg Br. 2006;88:727-729.

13. Legaye J, Duval-Beaupere G, Hecquet J, Marty C. Pelvic incidence: a fundamental pelvic parameter for three-dimensional regulation of spinal sagittal curves. Eur Spine J. 1998;7:99-103.

14. Leunig M, Podeszwa D, Beck M, Werlen S, Ganz R. Magnetic resonance arthrography of labral disorders in hips with dysplasia and impingement. Clin Orthop Relat Res. 2004;418:74-80.
15. Mast JW, Brunner RL, Zebrack J. Recognizing acetabular version in the radiographic presentation of hip dysplasia. Clin Orthop Relat Res. 2004;418:48-53.

16. Milone MT, Bedi A, Poultsides L, Magennis E, Byrd JW, Larson CM, Kelly BT. Novel CT-based three-dimensional software improves the characterization of cam morphology. Clin Orthop Relat Res. 2013;471:2484-2491.

17. Philippon MJ, Stubbs AJ, Schenker ML, Maxwell RB, Ganz R, Leunig M. Arthroscopic management of femoroacetabular impingement: osteoplasty technique and literature review. Am J Sports Med. 2007;35:1571-1580.

18. Reynolds D, Lucas J, Klaue K. Retroversion of the acetabulum. A cause of hip pain. J Bone Joint Surg Br. 1999;81:281-288.

19. Siebenrock KA, Kalbermatten DF, Ganz R. Effect of pelvic tilt on acetabular retroversion: a study of pelves from cadavers. Clin Orthop Relat Res. 2003;407:241-248.

20. Siebenrock KA, Schoeniger R, Ganz R. Anterior femoro-acetabular impingement due to acetabular retroversion. Treatment with periacetabular osteotomy. J Bone Joint Surg Am. 2003;85:278-286.

21. Tannast M, Murphy SB, Langlotz F, Anderson SE, Siebenrock KA. Estimation of pelvic tilt on anteroposterior X-rays-a comparison of six parameters. Skeletal Radiol. 2006;35:149-155.

22. Tannast M, Zheng G, Anderegg C, Burckhardt K, Langlotz F, Ganz R, Siebenrock KA. Tilt and rotation correction of acetabular version on pelvic radiographs. Clin Orthop Relat Res. 2005;438:182-190.

23. Wenger DR, Kishan S, Pring ME. Impingement and childhood hip disease. J Pediatr Orthop B. 2006;15:233-243.

24. Wiberg G. Studies on dysplastic acetabula and congenital subluxation of the hip: with special reference to the complication of osteoarthritis. Acta Chir Scand. 1939;58:7-38.

25. Pullen WM, Henebry A, Gaskill T. Variability of acetabular coverage between supine and weightbearing pelvic radiographs. Am J Sports Med. 2014 Sep 11 [Epub ahead of print].

26. Troelsen A, Jacobsen S, Romer L, Soballe K. Weightbearing anteroposterior pelvic radiographs are recommended in DDH assessment. Clin Orthop Relat Res. 2008;466:813-819. 